numerically equal. For if $\mathrm{T}_{k}=\mathrm{T}_{k+1}=-\mathrm{T}_{k+2}$, then $\frac{|x|}{|x|+1}(n+1)=k$ - and $\frac{|x|}{|x|-1}(n+1)=k+1$; solving these equations simultaneously we obtain the above values of $|x|$ and $n$. Thus

$$
(1+5)^{\frac{7}{5}}=1+7+7-7+14-\ldots
$$

Finally, for all values of $n$ the first term is the greatest if $|n x|<1$.

\title{
D. M. Y. Sommerville
}

An Experiment in Light.-Let ABCD be a horizontal section of a rectangular slab of glass. A pin is set up vertically at $P$, close to the face $A B$, or at a short distance from it. It is possible to see the pin $P$ through the glass if we look through the face CD; but the pin is invisible if we look through the face AD. If, however, we look through the face $\mathrm{CD}$, into the face $\mathrm{AD}$, we shall see an image of the pin in $A D$, which acts like a mirror. The experiment illustrates total reflection of light; the explanation is easy. Let $\mathrm{PO}$ be a ray of light which after passing into the glass will be incident on $\mathrm{AD}$ at $\mathrm{O}^{\prime}$. Let $\mathrm{NON}^{\prime}$ be the normal to $\mathrm{AB}$ at $\mathrm{O}$,

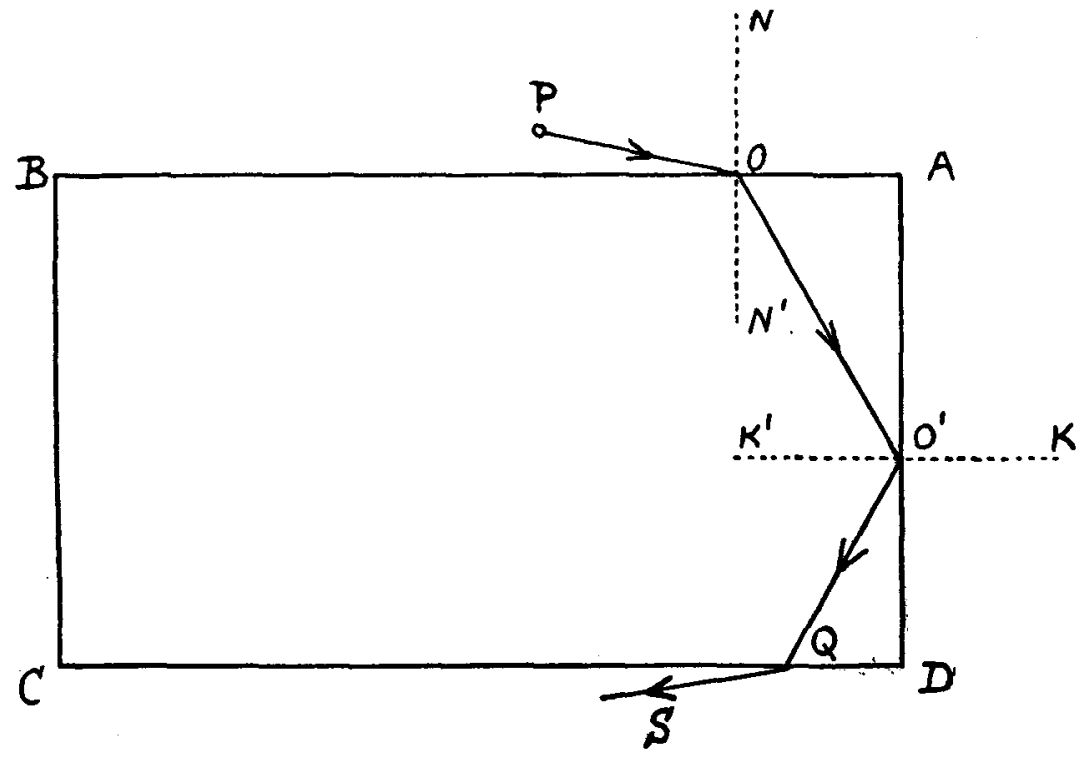


and $\mathrm{KO}^{\prime} \mathrm{K}^{\prime}$ be normal to $\mathrm{AD}$ at $\mathrm{O}^{\prime}$. The critical angle for glass being $41^{\circ} 45^{\prime}$ approximately,

the $\angle \mathrm{N}^{\prime} O \mathrm{O}^{\prime} \ngtr 41^{\circ} 45^{\prime}$;

$\therefore$ the $\angle O O^{\prime} \mathrm{K}^{\prime} \$ 48^{\circ} 15^{\prime}$;

i.e. the $\angle O O^{\prime} K^{\prime}>41^{\circ} 45^{\prime}$;

$\therefore$ the ray $O \mathrm{O}^{\prime}$ will not emerge through $\mathrm{AD}$, but will be reflected in the direction $O^{\prime} Q$, and will emerge at $Q$ in the direction QS.

\section{W. A. Lindsay}

Note on Fermat's Theorem.-The Theorem that $a^{n}-a$ is exactly divisible by $n$, if $n$ be a prime number, may be established as follows from the Binomial Theorem.

We have

$$
(a+b)^{n}=a^{n}+\mathrm{C}_{n}{ }^{1} a^{n-1} b+\mathrm{C}_{n}{ }^{2} a^{n-2} b^{2}+\ldots+\mathrm{C}_{n}{ }^{r} a^{n-r} b^{r}+\ldots+b^{n},
$$

where

$$
\mathrm{C}_{n}{ }^{r}=\frac{n(n-1)(n-2) \ldots(n-r+1)}{\underline{\underline{r}}} ;
$$

$$
\begin{aligned}
\therefore(a+b)^{n}-a^{n}-b^{n}=\mathrm{C}_{n}{ }^{1} a^{n-1} b+\mathrm{C}_{n}{ }^{2} a^{n-2} b^{2}+ & \ldots+\mathrm{C}_{n}{ }^{r} x^{n-r} b^{r} \\
& +\ldots+\mathrm{C}_{n}{ }^{n-1} a b^{n-1} .
\end{aligned}
$$

Now since $\mathrm{C}_{n}{ }^{r}$ is an integer, the product

$$
n(n-1)(n-2) \ldots(n-r+1)
$$

must contain as a factor the product $1.2 .3 \ldots r$; but if $n$ is a prime number it cannot contain as a factor any one of the integers $2,3,4, \ldots r$, each of which is $<n$;

$\therefore$ the product $\mid r$ must be contained in $(n-1)(n-2) \ldots(n-r+1)$, and $\therefore n$ is a factor of $\mathrm{C}_{n}{ }^{r}$.

This is true for all values of $r$ from 1 to $n-1$.

It follows from (1) that $(a+b)^{n}-a^{n}-b^{n}$ is exactly divisible by $n$.

Hence $(a+1)^{n}-a^{n}-1$ is exactly divisible by $n$.

i.e. $(a+1)^{n}-(a+1)-\left(a^{n}-a\right)$ is exactly divisible by $n$; which shows that if $a^{n}-a$ is exactly divisible by $n$ so will $(a+1)^{n}-(a+1)$.

Now from (2) it follows that $2^{n}-2$ is exactly divisible by $n$, (putting $a=1$ );

$\therefore 3^{n}-3$ is exactly divisible by $n$,

$\therefore 4^{n}-4$ is exactly divisible by $n$, and so on.

We might also reason as follows :-

To show that $a^{n}-a$ is exactly divisible by $n$, let $a=y+1$. 